\title{
Erratum to: Pontibacter locisalis Sy30T sp. nov. isolated from soil collected from an abandoned saltern
}

\author{
Yan-Xia Zhou $\cdot$ Zhi-Hong Xie • \\ Jin-Xin Zhao $\cdot$ Zong-Jun Du • Guan-Jun Chen
}

Published online: 1 July 2016

(C) Springer International Publishing Switzerland 2016

\section{Erratum to: Antonie van Leeuwenhoek (2016) 109:415-420 DOI 10.1007/s10482-016-0646-0}

Subsequent to the publication of the above paper, it has been noticed that some details need correcting, including the title and one of the type strain culture collection codes.

The correct title should read:

Pontibacter locisalis sp. nov. isolated from soil collected from an abandoned saltern

The last line of the Abstract should read:

The type strain is $\operatorname{Sy} 30^{\mathrm{T}}\left(=\mathrm{KCTC} 42498^{\mathrm{T}}=\mathrm{CCTCC}\right.$ AB 2015060 ${ }^{\mathrm{T}}$ ).

The corrected protologue is given below:

The online version of the original article can be found under doi:10.1007/s10482-016-0646-0.

Y.-X. Zhou · J.-X. Zhao · Z.-J. Du · G.-J. Chen $(\bowtie)$

College of Marine Science, Shandong University

at Weihai, Weihai 264209, China

e-mail: guanjun@sdu.edu.cn

Z.-H. Xie

Key Laboratory of Coastal Biology and Bioresource Utilization, Yantai Institute of Coastal Zone Research,

Chinese Academy of Sciences, Yantai 264003, China

\section{Z.-J. Du - G.-J. Chen}

State Key Laboratory of Microbial Technology,

Shandong University, Jinan 250100, China

\section{Description of Pontibacter locisalis sp. nov.}

Pontibacter locisalis (lo.ci.sa'lis. L. n. locus place, locality; L. gen. n. salis of salt; N.L. gen.

n. locisalis from a place of salt)

Cells are Gram-stain negative, non-motile, aerobic and rod-shaped with the size of $0.4-0.6 \mu \mathrm{m}$ in width and $2.0-7.0 \mu \mathrm{m}$ in length. Colonies are red, nonluminescent, and circular with entire edges on $2216 \mathrm{E}$ agar. Flexirubin-type pigments are absent. Growth occurs at $15-40{ }^{\circ} \mathrm{C}$ (optimum, $30-33{ }^{\circ} \mathrm{C}$ ), $\mathrm{pH}$ 6.5-9.0 (optimum, 7.0-8.0) and in presence $0.5-5.0 \%$ of $\mathrm{NaCl}$ (optimum, 2.0-2.5\%). Requires $\mathrm{NaCl}$ for growth. $\mathrm{Can}$ hydrolyze gelatin, aesculin, alginate and starch but not casein, cellulose and agar. Nitrate reduction, lysine decarboxylase, ornithine decarboxylase, $\mathrm{H}_{2} \mathrm{~S}$ production, citrate utilization, Voges-Proskauer reaction, indole production, glucose acidification, arginine dihydrolase and urease are negative. Positive for catalase, alkaline phosphatase, esterase (C4), esterase lipase (C8), leucine arylamidase, valine arylamidase, cystine arylamidase, trypsin, acid phosphatase, naphthol-AS-BI-phosphohydrolase and $\mathrm{N}$-acetyl- $\beta$-glucosaminidase but negative for oxidase, lipase (C14), $\alpha$-chymotrypsin, $\alpha$-galactosidase, $\beta$-galactosidase, $\beta$-glucuronidase, $\alpha$-glucosidase, $\beta$-glucosidase, $\alpha$-mannosidase and $\alpha$-fucosidase. Assimilates sucrose, D-lactose, D-glucose, D-galactose, D-fucose, L-rhamnose, D-melibiose, D-arabitol, D-trehalose but not D-mannose, L-alanine and sodium lactate. The predominant fatty acids are iso- $\mathrm{C}_{15: 0}$, and summed 
feature 4 (iso- $\mathrm{C}_{17: 1} \mathrm{I}$ and/or anteiso- $\mathrm{C}_{17: 1} \mathrm{~B}$ ). The major menaquinone is MK-7. The major polyamine is sym-homospermidine. The DNA G+C content of the type strain is $47.7 \mathrm{~mol} \%$. The polar lipids comprise major amounts of phosphatidylethanolamine, a phosphoaminolipid and two unidentified polar lipids, and minor amounts of two unidentified polar lipids and two phospholipids.
The type strain $\operatorname{Sy} 30^{\mathrm{T}}\left(=\mathrm{KCTC} 42498^{\mathrm{T}}=\mathrm{CCTCC}\right.$ AB $2015060^{\mathrm{T}}$ ), was isolated from dry soil of an abandoned marine solar saltern at Weihai, China. The GenBank accession number for the 16S rRNA gene sequence of strain $\mathrm{Sy} 30^{\mathrm{T}}$ is KR080555. 